\title{
Prediction of Postoperative Delirium after Gastrointestinal Surgery Using the Mie Constructional Apraxia Scale
}

\author{
Yosuke Tenpaku ${ }^{a}$ Masayuki Satoh ${ }^{b}$ Kenji Kato $^{c}$ Kazuhisa Fujinaga ${ }^{c}$ \\ Yuji Haruki $^{c}$ Hiroki Nakahashi ${ }^{c}$ Keisuke Morikawa ${ }^{a}$ Yasunori Imaoka ${ }^{a}$ \\ Hiroyuki Takemura $^{a}$ Hiroshi Tatsumi $^{d}$
}

aDepartment of Rehabilitation, Matsusaka Municipal Hospital, Matsusaka, Japan; ${ }^{b}$ Dementia Prevention and Therapeutics, Graduate School of Advanced Institute of Industrial Technology, Shinagawa, Japan; 'Department of Surgery, Matsusaka Municipal Hospital, Matsusaka, Japan; dDepartment of Health Science, Aichi Gakuin University, Nisshin, Japan

\section{Keywords}

Mie Constructional Apraxia Scale - Postoperative delirium . Gastroenterological surgery

\footnotetext{
Abstract

Background: Postoperative delirium (POD) is a transient postoperative complication that occurs after surgical procedures. Risk factors reported for POD include dementia and cognitive decline. The purpose of this study was to identify predictors of POD by examining the use of preoperative neuropsychological tests, including the Mie Constructional Apraxia Scale (MCAS), and patient background factors. Method: The study was performed as a retrospective cohort study. The subjects were 33 patients (mean age, $75.8 \pm 10.9$ years; male:female ratio, 26:7) who underwent gastrointestinal surgery at Matsusaka City Hospital between December 2019 and April 2021. Data were collected retrospectively from medical records. The study was started after receiving approval from the institution's ethics committee. The survey
}

items included general patient information, nutritional assessment, surgical information, and neuropsychological tests. Subjects were classified into 2 groups according to the presence or absence of POD. If a significant difference was observed between the 2 groups, the sensitivity, specificity, and area under the curve were calculated using a receiver operating characteristic (ROC) curve. Result: There were 10 patients in the POD group (male:female ratio, 6:4) and 23 patients in the non-POD group (20:3). The POD group had a shorter education history $(p=0.047)$ and significantly higher MCAS scores $(p=0.007)$ than the non-POD group. The ROC curve showed a sensitivity of $90 \%$, a specificity of $69 \%$, and an area under the curve of 0.798 when the MCAS cutoff value was set at 3 points. Conclusion: Preoperative MCAS results were capable of predicting the occurrence of POD after gastrointestinal surgery. In addition, a relatively short education background was also considered a risk factor for POD.

(C) 2021 The Author(s)

Published by S. Karger AG, Basel karger@karger.com www.karger.com/dee

Karger $\stackrel{\text { ' }}{5}$

BOPEN ACCESS
(C) 2021 The Author(s)

Published by S. Karger AG, Basel

This is an Open Access article licensed under the Creative Common Attribution-NonCommercial-4.0 International License (CC BY-NC) (http://www.karger.com/Services/OpenAccessLicense), applicable to the online version of the article only. Usage and distribution for commercial purposes requires written permission.
Correspondence to:

Yosuke Tenpaku,palu109291@gmail.com 


\section{Introduction}

Postoperative delirium (POD) is an acute brain dysfunction that occurs after surgery. POD causes impairment of consciousness, cognitive function, perception, and attention. It usually develops acutely during the early postoperative period extending to a few days after surgery [1]. POD in the elderly has various negative effects, such as prolonged hospitalization and a decline in physical and cognitive functions [1]. The impact of POD on the elderly is significant and an important issue.

Delirium has been reported to be a risk factor for dementia and cognitive decline in the elderly [2]. POD has also been reported to be associated with the possibility of background dementia [3] and even increased mortality [4]. The effects may persist even after the symptoms disappear. There are various risk factors for POD. Risk factors include age, education, cognitive decline, visual or hearing impairment, emergency hospitalization or surgery, alcoholism, drug abuse, electrolyte abnormalities, chronic kidney disease, low nutrition, and frailty [5]. A variety of risk factors are considered to be capable of predicting POD before surgery. However, the performance of lengthy evaluations and tests prior to surgery can be difficult.

Cognitive decline is one of the risk factors for the development of POD. Previous studies using neuropsychological tests have been scattered. One study used the Hasegawa's Dementia Scale-Revised (HDS-R) [6] and the Kana pick-out test [7], which are widely used in Japan for screening of cognitive decline. In that study, a low score on the Kana pick-out test was reported to be a risk factor for POD [8]. Another study used the Mini-Mental State Examination (MMSE) [9], which is widely used around the world [10]. In that study, preoperative MMSE scores below the cutoff value (cutoff, 24 points) were reported to be a risk factor for POD.

The Necker Cube Copying test is a neuropsychological test that is rated according to the shape that is drawn. The Necker Cube Copying test is useful for detecting constructional apraxia, which is one of the higher brain dysfunctions that can occur after cerebrovascular disease [11]. In addition, previous studies have reported that the Necker Cube Copying test is useful for detecting cognitive decline [12]. In 2016, Satoh et al. [13] improved the assessment of the Necker Cube Copying test and created the Mie Constructional Apraxia Scale (MCAS) (Fig. 1). The MCAS differs from previous methods because it evaluates not only the shape but also the process and time required for copying. The MCAS assessment is easy and takes only a minute or two to complete. Compared with conventional scoring methods, this method is more sensitive for detecting constructional apraxia and cognitive decline. Although cognitive decline is known to be a risk factor of POD, the performance of examination which requires a long time is difficult prior to surgery. If a test that can be easily performed at bedside and is sensitive to the development of POD, this test could be useful for preventing the occurrence of POD and planning its care. In this study, we examined preoperative factors capable of predicting the onset of POD in a retrospective manner based on medical records.

\section{Materials and Methods}

The study design was a single-center retrospective observational study. The subjects were patients who had been admitted to our Gastroenterology Department for surgical resection between December 2019 and April 2021. Forty-three patients had completed a preoperative intervention performed by a speech-language-hearing therapist (ST). Thirty-three study participants (mean age, 75.8 \pm 10.9 years; male:female ratio, 26:7) did not meet any of the following exclusion criteria: (1) MMSE $\leq 23$ points; (2) active cerebrovascular disease, neurological disease, and a history of dementia; (3) performed as emergency surgery; and (4) rejection. The 33 participants were classified into 2 groups according to the presence or absence of POD (Fig. 2).

The survey items included general patient information such as age, sex, BMI, number of days in the High Care Unit, education history, and medical history. As neuropsychological tests, the MMSE and MCAS were performed. Surgical information was collected from the surgical and medical records of the surgeons. Surgical information was evaluated for preoperative systolic blood pressure, diastolic blood pressure, pulse, operative time, operative procedure (open/laparoscopic), presence of complications, C-reactive protein measured on days 1 and 3 postoperatively, operative site, and malignancy. The Geriatric Nutritional Risk Index (GNRI) [14] was evaluated as a nutritional assessment. The GNRI was calculated using the formula $(14.89 \times \mathrm{Alb})+41.7 \times(\mathrm{BW} / \mathrm{IBW})$. Using the collected data, the study was started after receiving approval from the Ethics Committee of Matsusaka Municipal Hospital.

The MCAS is scored using a 12-point scale, with a lower score indicating a lower degree of disability. The time required for the test was also measured, and the shorter the time required, the lower the degree of impairment. The MCAS is simple to perform and can be easily assessed in clinical settings even by nonspecialists of neurology or psychiatry. Assessment of the drawing process can also detect mild constructional apraxia caused by various cognitive deficits, such as visuospatial, executive, and attentional processing.

The definition of POD used in this study was the same as that used in previous studies [15]. A patient was diagnosed as having POD when he/she showed one or more following symptom(s) temporarily after surgery: (1) wandering, (2) self-removal of injection routes, (3) visual hallucinations, (4) auditory hallucinations, and (5) abnormal excitement. These symptoms were confirmed by several persons including physical therapists, occupational thera- 


\begin{tabular}{|c|c|c|c|}
\hline \multirow[b]{2}{*}{ Name: } & \multicolumn{3}{|c|}{ Date: } \\
\hline & age: & y.o. & sex: \\
\hline Order & Check points & Yes & No \\
\hline 1 & The subject did NOT complete the front or the upper face. & 1 & 0 \\
\hline 2 & Closing-in phenomenon was observed. & 1 & 0 \\
\hline 3 & The subject drew inside the lines, before he/she completed the external form. & 1 & 0 \\
\hline 4 & $\begin{array}{l}\text { Longitudinal sides were of different length (including those that the subject drew } \\
\text { more than once). }(* 1)\end{array}$ & 1 & 0 \\
\hline 5 & $\begin{array}{l}\text { Horizontal sides were of different length (including those that the subject drew } \\
\text { more than once). }(* 2)\end{array}$ & 1 & 0 \\
\hline 6 & The subject drew an outline which extended over several faces. & 1 & 0 \\
\hline 7 & $\begin{array}{l}\text { The subject hesitated and stopped drawing at the cross-points of inner and } \\
\text { anterior sides. }(* 3)\end{array}$ & 1 & 0 \\
\hline 8 & The subject could not complete his/her drawing, and drew more than once. $(* 4)$ & 1 & 0 \\
\hline 9 & Presence of undrawn side(s) (including when a side was incomplete). & 1 & 0 \\
\hline 10 & $\begin{array}{l}\text { If the side was shifted from the apex but the subject did NOT correct it } \\
\text { (especially the right bottom apex). }\end{array}$ & 1 & 0 \\
\hline 11 & Interruption beyond five seconds. & 1 & 0 \\
\hline 12 & Subjects needed more than one minute to finish the drawing. & 1 & 0 \\
\hline & Total & & $/ 12$ \\
\hline & Time & $\min$ & sec \\
\hline
\end{tabular}

Instruction: Copy this figure as correctly as possible.

We would like to record your drawing by video camera for later assessment.

Evaluation of the shape according to the Strub \& Black score (2000)

Examples of drawings: Normal pattern

The subject drew the inner sides after he/she completed the external form of the cube.

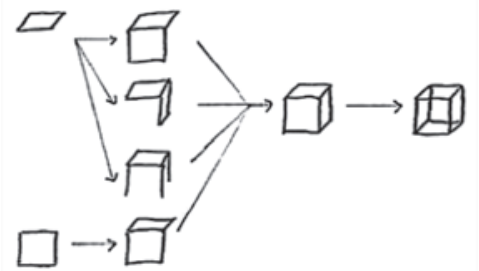

Fig. 1. Mie Constructional Apraxia Scale.

pists, and ST. In addition, the contents were discussed in a conference with the ward nurses and were confirmed by the attending physician. The above process was used to diagnose POD.

For the statistical analysis, group comparisons were examined using the $\chi^{2}$ test and the Mann-Whitney U test. If significant differences in the neuropsychological tests were observed between the 2 groups, the sensitivity, specificity, and area under the curve (AUC) were determined using the receiver operating characteristic curve (ROC curve). We used SPSS Ver. 25 (IBM, Chicago, IL, USA) as the statistical analysis software.
The patients' data were not used for any purposes other than the presently reported research. All personal data were processed quantitatively in a manner such that individuals could not be identified. This study was conducted after obtaining approval from the Ethics Committee of Matsusaka Municipal Hospital (Approval No. J-131-210514-1-1), and it was performed in accordance with the Helsinki Declaration of 1975. In addition, this study was conducted in accordance with the Ethical Guidelines for Medical and Health Research Involving Human Subjects of 2021. 
Fig. 2. Flowchart.

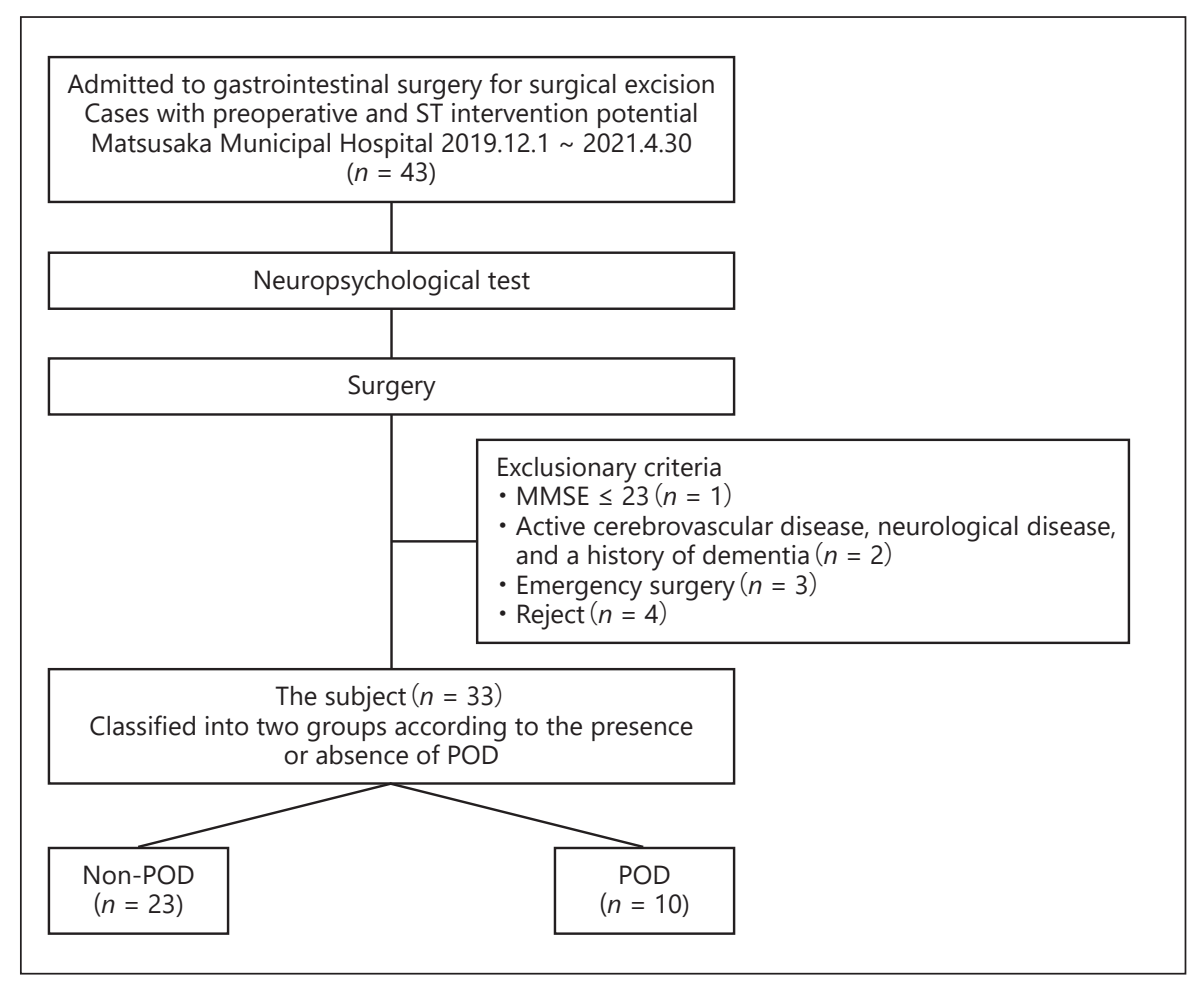

\section{Results}

After surgery, wandering was observed in 2 cases, selfremoval of injection routes was observed in 5 cases, visual and auditory hallucinations were observed in 7 cases, and abnormal excitement was observed in 8 cases (some patients exhibited multiple features). POD was confirmed in 10 cases. POD appeared on the first postoperative day in 8 patients and on the second postoperative day in 2 patients. All the patients' conditions had improved by postoperative day 6 (mean duration of POD, $2.3 \pm 1.6$ days).

Comparisons were made between the POD group and the non-POD group. The results showed that the POD group had a significantly shorter education history $(p=$ 0.047 ). In addition, there was a tendency for patients in the POD group to be female $(p=0.082)$ and to have a history of dyslipidemia $(p=0.053)$. No significant differences in age; hospital stay; BMI; length of high care unit stay; presence of cerebrovascular disease, cardiac disease, hepatic disease, renal disease, respiratory disease, or diabetes; history of hypertension; smoking or alcohol consumption; or GNRI were seen between the 2 groups (Table 1).

In terms of surgical information, no significant differences in preoperative systolic blood pressure, diastolic blood pressure, pulse, operative time, operative tech- nique (laparotomy or laparoscopy), postoperative complications, postoperative CRP level, or operative site were seen. The results of the neuropsychological tests showed no significant differences in the MMSE scores or the MCAS time. However, a significant difference in MCAS scores was seen between the 2 groups ( $p=0.007$ ) (Table 2). The ROC curve was examined for the MCAS scores. As a result, the sensitivity, specificity, and AUC were calculated to be $90 \%, 69 \%$, and 0.798 , respectively, for an MCAS cutoff value of 3 (Fig. 3).

\section{Discussion}

The findings of this study can be summarized as follows: (1) the MCAS score was identified as a factor that could predict the occurrence of POD, (2) the MCAS cutoff value for predicting POD was calculated to be 3 , and (3) a shorter education history was extracted as a risk factor for POD. The preoperative MCAS score was shown to be a predictor of the development of POD. Baranowski et al. [16] administered a test of constructional apraxia that could be easily administered at bedside to psychiatric inpatients. As a result, they reported that the presence of dysgraphia and constructional apraxia was predictive of the occurrence of delirium. Cognitive decline is a risk factor for POD. Oldham 
Table 1. Results of comparison of general patient information, medical history, and nutritional assessment in nonPOD and POD groups

\begin{tabular}{lllll}
\hline & Overall $(n=33)$ & Non-POD $(n=23)$ & POD $(n=10)$ & $p$ value \\
\hline General patient information & & & & \\
Age, years & $75.8 \pm 10.9$ & $73.9 \pm 12.4$ & $80.3 \pm 3.7$ & 0.188 \\
Gender (M/F) & $26 / 7$ & $20 / 3$ & $6 / 4$ & 0.082 \\
Hospital days & $31.7 \pm 21.7$ & $30.8 \pm 22.3$ & $33.7 \pm 21.2$ & 0.570 \\
BMI, kg/m & $21.9 \pm 3.0$ & $21.8 \pm 2.9$ & $22.1 \pm 3.3$ & 0.969 \\
Education, years & $11.0 \pm 2.3$ & $11.5 \pm 2.4$ & $9.9 \pm 1.4$ & 0.047 \\
HCU in room, days & $1.0 \pm 0.0$ & $1.0 \pm 0.0$ & $1.0 \pm 0.0$ & 1.000 \\
Past history (people), $n$ (\%) & & & & 0.361 \\
Cerebrovascular disease & $4(12)$ & $2(9)$ & $2(20)$ & 0.592 \\
Cardiac disease & $11(33)$ & $7(30)$ & $4(40)$ & 1.000 \\
Liver disease & $0(0.0)$ & $0(0.0)$ & $0(0.0)$ & 0.503 \\
Renal disease & $1(3)$ & $1(4)$ & $3(30)$ & 0.466 \\
Respiratory disease & $10(30)$ & $7(30)$ & $3(30)$ & 0.789 \\
Diabetes & $11(33)$ & $8(35)$ & $6(60)$ & 0.178 \\
Hypertension & $14(42)$ & $10(43)$ & $6(80)$ & 0.053 \\
Dyslipidemia & $18(55)$ & $15(65)$ & $5(60)$ & 0.280 \\
Smoking history & $21(64)$ & $10(43)$ & & 0.730 \\
Drinking history & $15(45)$ & $94.0 \pm 9.2$ & $94.7 \pm 11.2$ & 0.891 \\
Nutritional assessment & $94.2 \pm 9.7$ & &
\end{tabular}

BMI, body mass index; $\mathrm{HCU}$, high care unit; GNRI, geriatric nutritional risk index.

Table 2. Comparative results of surgical information and neuropsychological tests in non-POD and POD groups

\begin{tabular}{|c|c|c|c|c|}
\hline & Overall $(n=33)$ & Non-POD $(n=23)$ & $\operatorname{POD}(n=10)$ & $p$ value \\
\hline \multicolumn{5}{|l|}{ Surgery information } \\
\hline Operating time, minutes & $328.7 \pm 174.5$ & $311.7 \pm 157.2$ & $367.8 \pm 212.6$ & 0.969 \\
\hline Procedure (open/laparoscopic) & $23 / 10$ & $17 / 6$ & $6 / 4$ & 0.424 \\
\hline Complications (have/none) & $11 / 22$ & $7 / 16$ & $4 / 6$ & 0.592 \\
\hline Postoperative day $1 \mathrm{CRP}, \mathrm{mg} / \mathrm{L}$ & $7.8 \pm 3.8$ & $7.8 \pm 3.7$ & $7.9 \pm 4.1$ & 0.769 \\
\hline Postoperative day $3 \mathrm{CRP}, \mathrm{mg} / \mathrm{L}$ & $11.2 \pm 6.1$ & $11.1 \pm 6.5$ & $11.3 \pm 5.4$ & 1.000 \\
\hline Systolic blood pressure, $\mathrm{mm} \mathrm{Hg}$ & $115.5 \pm 15.0$ & $113.0 \pm 13.2$ & $121.5 \pm 17.8$ & 0.308 \\
\hline Diastolic blood pressure, $\mathrm{mm} \mathrm{Hg}$ & $68.1 \pm 9.9$ & $66.8 \pm 9.3$ & $71.2 \pm 11.2$ & 0.264 \\
\hline Pulse rate (times) & $67.9 \pm 9.5$ & $68.4 \pm 9.2$ & $66.9 \pm 10.1$ & 0.596 \\
\hline \multicolumn{5}{|l|}{ Surgical site (people), $n$ (\%) } \\
\hline Stomach & $11(33)$ & $6(26)$ & $5(50)$ & 0.181 \\
\hline Pancreas & $8(24)$ & $5(22)$ & $3(30)$ & 0.611 \\
\hline Colon & $12(36)$ & $10(43)$ & $2(20)$ & 0.198 \\
\hline Others & $2(6)$ & $2(9)$ & $0(0)$ & 0.336 \\
\hline Malignant tumor & $30(91)$ & $20(87)$ & $10(100)$ & 0.231 \\
\hline \multicolumn{5}{|l|}{ Neuropsychological examination } \\
\hline MMSE (point) & $27.4 \pm 1.9$ & $27.5 \pm 1.8$ & $27.2 \pm 2.1$ & 0.795 \\
\hline MCAS (point) & $3.0 \pm 2.2$ & $2.4 \pm 2.2$ & $4.4 \pm 1.6$ & 0.007 \\
\hline MCAS (time) & $40.0 \pm 16.3$ & $37.7 \pm 16.4$ & $45.3 \pm 13.9$ & 0.227 \\
\hline
\end{tabular}

MMSE, Mini-Mental State Examination; MCAS, Mie Constructional Apraxia Scale. 


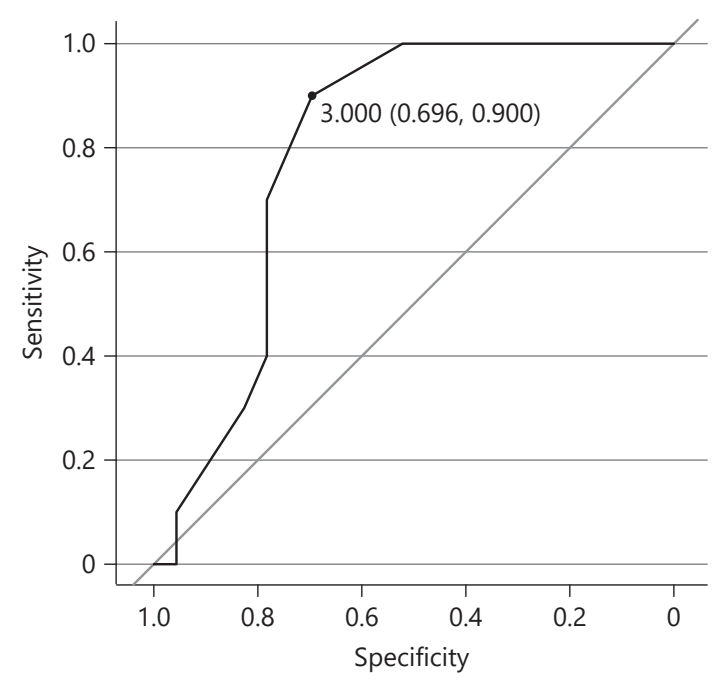

Fig. 3. ROC curve results for MCAS point. AUC: 0.798, sensitivity: $90 \%$, specificity: $69 \%$, and cutoff: 3 . ROC curve, receiver operating characteristic curve; AUC, area under the curve.

et al. [17] reported that mild cognitive impairment (MCI) was extracted as a risk for developing POD after coronary artery bypass surgery. Shimada et al. [12] also reported that the Necker Cube Copying test was useful for predicting MCI. MCAS has been reported to be correlated with intelligence, memory, and frontal lobe function tests [13]. These previous studies suggest that a preoperative MCAS could be used to assess mild cognitive decline that is not yet apparent in daily living. Therefore, application of the MCAS might be useful for predicting the occurrence of POD.

In the present study, the MCAS cutoff value for predicting POD was 3 points. Thirteen patients were below the cutoff value (delirium group, 8; nondelirium group, 5). Satoh et al. [13] previously reported that the MCAS cutoff for determining constructional apraxia was between 2 and 3 points. Thus, the results of the present study were consistent with those of previous studies.

As far as we know, the only study to investigate the relationship between POD and brain activity was reported by Fong et al. [18]. They used SPECT to evaluate cerebral blood flow in 22 patients with hypoactive delirium. The results showed that all the patients had decreased cortical blood flow, and one of them had both right occipital hypoperfusion and left frontal hyperperfusion. The Necker Cube Copying test is based on the functional associations of the occipital, parietal, and frontal lobes. The MCAS can assess frontal lobe functions, such as executive functions, by evaluating the drawing process. The possibility that the MCAS, which scores these functions, showed a significant association was considered.

The results of the present study examining general patient information showed that the delirium group had a significantly shorter education history. Many reports have described an association between delirium and a shorter education history. Sprung et al. [19] conducted a factor analysis of POD in patients who underwent various surgeries. The study reported that a shorter education history was a risk factor for POD. Martins et al. [20] also investigated delirium in patients admitted to the ICU. Their results indicated that a short education history was also a risk factor of POD. The present study was congruent with the results of previous studies mentioned above. The present study had some limitations. First, this was a retrospective cohort study. Second, the number of eligible patients was small. In the future, prospective studies are needed to determine whether MCAS can really predict POD. Significant trends for characteristics that have not been previously reported were observed in the POD group, such as a higher incidence among women and the presence of dyslipidemia. It is possible that there was a case bias in the results of this study.

It is also possible that the POD occurred in patients who were already suspected of having cognitive decline before the surgery. We cannot deny the possibility of various biases.

In future studies, larger numbers of cases and the removal of possible biases will be needed. Third, the current study did not examine brain imaging and did not rigorously rule out the possible involvement of intracranial disease related to the development of POD. Brain imaging studies would provide a more reliable diagnosis of POD and would enable comparisons with the results of neuropsychological tests. In this study, however, all the patients' POD symptoms improved, and it is unlikely that they had organic intracranial diseases. Finally, neuropsychological tests other than the MMSE and MCAS were not available in this study. The results of this study suggest that neuropsychological tests that assess frontal lobe function and mild cognitive decline may also be able to predict POD. A larger cohort study and the comparison of the results of MCAS with another neuropsychological test are needed. The usefulness of POD prediction should then be examined in more detail.

Despite these limitations, the present study indicates that preoperative MCAS may be useful for predicting the development of POD. The evaluations and tests whose performances need much time are difficult to be performed before surgery. The MCAS, however, can be eas- 
ily performed at bedside and takes only a few minutes to complete. POD is correlated with a variety of negative factors, including postoperative mortality, functional decline, increased hospital stays, and higher medical costs [21]. Delirium also increases the burden on hospital staff [22]. If POD can be predicted simply by applying the MCAS before surgery, it might be possible to prevent POD or to take appropriate care. Given these advantages, the findings of this study are notable.

\section{Conclusion}

This study retrospectively examined the prediction of POD based on medical records. The results showed that the MCAS might be useful for predicting POD. In the future, a prospective study examining a larger number of cases is needed.

\section{Statement of Ethics}

Before conducting this study, we obtained approval from the Ethics Committee of Matsusaka City Hospital (Approval No. J-131-210514-1-1). This study is a retrospective study. It was difficult to obtain verbal approval for the patients. This consent protocol was reviewed and the need for written and informed consent was waived by the Ethics Committee of Matsusaka City Hospital (Approval No. J-131-210514-1-1). The research description was posted on the hospital website for public awareness. In addition, explanatory messages regarding the protection of personal information were posted in the rehabilitation room to make people aware of the issue. This study was conducted in accordance with the Helsinki Declaration of 1975.

\section{Conflict of Interest Statement}

The authors have no conflicts of interest to declare.

\section{Funding Sources}

The authors have no funding sources to declare.

\section{Author Contributions}

All authors meet the criteria of the ICMJE Criteria for Authorship. Masayuki Satoh, Keisuke Morikawa, Yasunori Imaoka, and Hiroyuki Takemura contributed to conception and design of the study. Kenji Kato, Kazuhisa Fujinaga, Yuji Haruki, and Hiroki Nakahashi contributed to acquisition of data. Masayuki Satoh, Keisuke Morikawa, Yasunori Imaoka, Hiroyuki Takemura, Kenji Kato, Kazuhisa Fujinaga, Yuji Haruki, Hiroki Nakahashi, and Hiroshi Tatsumi contributed to analysis and/or interpretation of data. Masayuki Satoh and Hiroshi Tatsumi contributed to drafting the manuscript. Masayuki Satoh, Keisuke Morikawa, Yasunori Imaoka, Hiroyuki Takemura, Kenji Kato, Kazuhisa Fujinaga, Yuji Haruki, Hiroki Nakahashi, and Hiroshi Tatsumi contributed to revising the manuscript critically for important intellectual content. Masayuki Satoh, Keisuke Morikawa, Yasunori Imaoka, Hiroyuki Takemura, Kenji Kato, Kazuhisa Fujinaga, Yuji Haruki, Hiroki Nakahashi, and Hiroshi Tatsumi contributed to approval of the version of the manuscript to be published.

\section{Data Availability Statement}

This study was based on a retrospective collection of medical records. Therefore, there is a risk that the data may contain personal information. For these reasons, we cannot release the data.

\section{References}

1 Martins S, Fernandes L. Delirium in elderly people: a review. Front Neurol. 2012 Jun 19;3: 101.

2 Davis DH, Muniz Terrera G, Keage H, Rahkonen T, Oinas M, Matthews FE, et al. Delirium is a strong risk factor for dementia in the oldest-old: a population-based cohort study. Brain. 2012;135:2809-16.

3 Kat MG, Vreeswijk R, de Jonghe JF, van der Ploeg T, van Gool WA, Eikelenboom P, et al. Long-term cognitive outcome of delirium in elderly hip surgery patients. A prospective matched controlled study over two and a half years. Dement Geriatr Cogn Disord. 2008;26: $1-8$.

4 Gottesman RF, Grega MA, Bailey MM, Pham LD, Zeger SL, Baumgartner WA, et al. Delirium after coronary artery bypass graft surgery and late mortality. Ann Neurol. 2010;67:33844.
5 Needham MJ, Webb CE, Bryden DC. Postoperative cognitive dysfunction and dementia: what we need to know and do. Br J Anaesth. 2017;119:1115-25.

6 Hasegawa K, Inoue K, Moriya K. [An investigation of dementia rating scale for elderly]. Seishin Igaku. 1974;16:965-9.

7 Kaneko M. Dementia and frontal lobe function. High Brain Funct Res. 1990;10(2):12731.

8 Morimoto Y, Yoshimura M, Utada K, Setoyama K, Matsumoto M, Sakabe T. Prediction of postoperative delirium after abdominal surgery in the elderly. J Anesth. 2009;23:51-6.

9 Folstein MF, Folstein SE, McHugh PR. "Minimental state." A practical method for grading the cognitive state of patients for the clinician. J Psychiatr Res. 1975;12:189-98.
10 Böhner H, Hummel TC, Habel U, Miller C, Reinbott S, Yang Q, et al. Predicting delirium after vascular surgery: a model based on pre- and intraoperative data. Ann Surg. 2003;238:149-56.

11 Caminiti R, Chafee MV, Battaglia-Mayer A, Averbeck BB, Crowe DA, Georgopoulos AP. Understanding the parietal lobe syndrome from a neurophysiological and evolutionary perspective. Eur J Neurosci. 2010;31:2320-40.

12 Shimada Y, Meguro K, Kasai M, Shimada M, Ishii $\mathrm{H}$, Yamaguchi $\mathrm{S}$, et al. Necker cube copying ability in normal elderly and Alzheimer's disease. A community-based study: the Tajiri project. Psychogeriatrics. 2006;6(1):4-9.

13 Satoh M, Mori C, Matsuda K, Ueda Y, Tabei $\mathrm{KI}$, Kida $\mathrm{H}$, et al. Improved Necker cube drawing-based assessment battery for constructional apraxia: the Mie Constructional apraxia scale (MCAS). Dement Geriatr Cogn Dis Extra. 2016 Sep 23;6:424-36. 
14 Cereda E, Pedrolli C, Zagami A, Vanotti A, Piffer S, Opizzi A, et al. Nutritional screening and mortality in newly institutionalised elderly: a comparison between the geriatric nutritional risk index and the mini nutritional assessment. Clin Nutr. 2011;30:793-8.

15 Marcantonio ER, Goldman L, Mangione CM, Ludwig LE, Muraca B, Haslauer CM, et al. A clinical prediction rule for delirium after elective noncardiac surgery. JAMA. 1994 Jan 12; 271:134-9.

16 Baranowski SL, Patten SB. The predictive value of dysgraphia and constructional apraxia for delirium in psychiatric inpatients. Can J Psychiatry. 2000;45:75-8.
17 Oldham MA, Hawkins KA, Yuh DD, Dewar ML, Darr UM, Lysyy T, et al. Cognitive and functional status predictors of delirium and delirium severity after coronary artery bypass graft surgery: an interim analysis of the neuropsychiatric outcomes after heart surgery study. Int Psychogeriatr. 2015;27:1929-38.

18 Fong TG, Bogardus ST Jr, Daftary A, Auerbach E, Blumenfeld H, Modur S, et al. Cerebral perfusion changes in older delirious patients using 99mTc HMPAO SPECT. J Gerontol A Biol Sci Med Sci. 2006;61:1294-9.

19 Sprung J, Roberts RO, Weingarten TN, Nunes Cavalcante A, Knopman DS, Petersen RC, et al. Postoperative delirium in elderly patients is associated with subsequent cognitive impairment. Br J Anaesth. 2017;119:316-23.
20 Martins S, Paiva JA, Simões MR, Fernandes L. Delirium in elderly patients: association with educational attainment. Acta Neuropsychiatr. 2017;29:95-101.

21 Khadka J, McAlinden C, Pesudovs K. Cognitive trajectories after postoperative delirium. N Engl J Med. 2012;367:1164-5.

22 Schmitt EM, Gallagher J, Albuquerque A, Tabloski P, Lee HJ, Gleason L, et al. Perspectives on the delirium experience and its burden: common themes among older patients, their family caregivers, and nurses. Gerontologist. 2019;59:327-37. 